\title{
Evaluation of the relationship between cholesterol levels and multiple sclerosis disease activity
}

\author{
Meral Seferoğlu ${ }^{1}$, Nizameddin Koca ${ }^{\circledR} \odot$
}

${ }^{1}$ Department of Neurology, University of Health Sciences, Bursa Yüksek Ihtisas Training and Research Hospital, Bursa, Turkey

${ }^{2}$ Department of Internal Medicine, University of Health Sciences, Bursa Yüksek İhtisas Training and Research Hospital, Bursa, Turkey

\begin{abstract}
Objectives: Multiple sclerosis (MS) is an immune-mediated, inflammatory, demyelinating, neurodegenerative disease of the central nervous system affecting young adults. Cholesterol and lipids are essential components of nerve cells and are abundant in the myelin sheath. In this study, we aimed to investigate the relationship between plasma cholesterol levels and severity of the disease and lesion burden in cranial magnetic resonance imaging.

Methods: A total of 70 patients (22 males and 48 females) with the diagnosis of MS were included in the study. Age, gender, duration of disease, Expanded Disability Status Scale (EDSS) scores, total number of relapses since diagnosis, current treatment and lipid levels of all participants were recorded. The patients were grouped according to EDSS scores, total number of relapses and number of lesions observed in cranial MRI and the data were compared among the groups.

Results: The mean age was $38.62 \pm 9.94$ years and the duration of the disease was $7.50 \pm 5.88$ years. The mean EDSS score was $2.80 \pm 1.69$. Total cholesterol, triglyceride and LDL levels were found to be significantly higher in the group with more than 3 relapses. Patients with $>9$ lesions were older and EDSS scores were higher. In correlation analysis; there was a significant positive correlation between total cholesterol and LDL levels and EDSS scores and disease duration.

Conclusions: We found that the number of relapses, disease duration, and EDSS scores were significantly correlated with cholesterol levels. The changes in plasma cholesterol levels which are easily accessible laboratory tests may provide insight into MS disease activity and progression.

Keywords: Multiple sclerosis, total cholesterol, low-density lipoprotein, high-density lipoprotein, triglycerides
\end{abstract}

$\mathrm{M}$ ultiple sclerosis (MS) is an immune-mediated, inflammatory, demyelinating and neurodegenerative disease of the central nervous system affecting young adults. Myelin damage, loss of myelin and oligodendrocytes, and reactive astrogliosis are considered as the underlying processes in the complex pathogenesis. Clinical findings depend on the damaged area in the brain and severity of injury. Serious disability associated with these complex pathological features and unpredictable course of disease leads to difficulties with the follow-up of the patients. Therefore, easily accessible biomarkers associated with disease activity and outcomes are needed to improve our understanding of specific disease processes in MS [1].

Cholesterol and lipids are essential components of nerve cells. In particular, myelin synthesis and stabi- 
lization are a cholesterol-dependent process. Several studies have reported that high plasma cholesterol levels were associated with clinical deterioration in dementia and MS. Thereafter, some studies have begun to investigate the travel of cholesterol between the plasma and central nervous system $[2,3]$. Changes in the cholesterol levels, which are abundant in the myelin sheath, may have some consequences in the course of the disease. There is no clear information confirming this relationship.

In this study, we aimed to investigate the correlation between cholesterol levels and disease severity, recurrence of relapses and lesion burden in cranial magnetic resonance imaging (MRI) in MS patients.

\section{METHODS}

A total of 70 patients ( 22 males and 48 females) who were followed-up with the diagnosis of MS according to the $2010 \mathrm{Mc}$-Donalds diagnostic criteria and admitted to our MS outpatient clinic between August 2017 and August 2019 were included in the study. Patients were between 18-65 years old and had no history of relapses and steroid treatment in the last 3 months. Patients who were diagnosed with diabetes mellitus, lipid metabolism disorder, thyroid disease, liver and renal disease and with any treatment for these diagnoses were excluded. Ethical approval (protocol number: 2011-KAEK-25 2019 / 10-11) was granted from the local ethical committee and the study was conducted according to declaration of Helsinki.

Age, gender, duration of disease, Expanded Disability Status Scale (EDSS) scores, total number of relapses since diagnosis, current treatment and lipid levels of all participants were recorded. The patients were grouped according to EDSS scores, total number of relapses and number of lesions observed in cranial MRI and data were compared among the groups.

\section{Statistical Analysis}

SPSS 22 software was used for statistical analysis. Independent sample t-test was used for descriptive statistical methods (mean, standard deviation) and comparison of the two groups. Chi-square test was used for the comparison of parametric data. The results were evaluated at $p<0.05$ level of significance.

\section{RESULTS}

The mean age, disease duration and EDSS scores of the participants were $38.62 \pm 9.94$ years, $7.50 \pm 5.88$ years and $2.80 \pm 1.69$, respectively. Twenty-seven patients had more than 3 relapses and 47 patients had $>9$ lesions in cranial MRI. The general characteristics of the participants are shown in Table 1.

The demographic data, total cholesterol, LDL, and triglyceride levels of the patients were compared between 2 groups (group 1: $\leq 3$ relapses, group 2: $>3$ relapses).There was no significant difference between the groups in terms of age and gender, while the duration of disease was higher in patients with relapses $>3$ (Table 2). While the total cholesterol, triglyceride and LDL levels were found to be significantly higher in group $2(p<0.01, p=0.045$, and $p=0.001$, respectively), no significant differences were detected with HDL cholesterol levels between the groups ( $p=$ $0.573)$. The duration of the disease was significantly longer in group $2(p=0.007)$. No significant differences were found among the EDSS scores and cranial MRI lesion counts.

The patients were divided into two groups as $\leq 9$

\section{Table 1. General characteristics of study \\ population}

\begin{tabular}{|c|c|}
\hline Male/ female & $22 / 48$ \\
\hline Age, years & $38.62 \pm 9.94$ \\
\hline Disease duration, years & $7.50 \pm 5.88$ \\
\hline Total cholesterol, mg/dL & $198.12 \pm 59.29$ \\
\hline Triglycerides, $\mathrm{mg} / \mathrm{dL}$ & $134.16 \pm 85.45$ \\
\hline HDL, mg/dL & $52.17 \pm 15.31$ \\
\hline $\mathrm{LDL}, \mathrm{mg} / \mathrm{dL}$ & $116.69 \pm 46.65$ \\
\hline EDSS score & $2.8 \pm 1.69$ \\
\hline \multicolumn{2}{|l|}{ Number of lesions } \\
\hline 1-9 Lesions & 23 \\
\hline$>9$ Lesions & 47 \\
\hline \multicolumn{2}{|l|}{ Number of relapses } \\
\hline$\leq 3$ relapses & 43 \\
\hline$>3$ relapses & 27 \\
\hline
\end{tabular}

Data are shown mean \pm standard deviation or number. EDSS $=$ Expanded Disability Status Scale, LDL = low-density lipoprotein, $\mathrm{HDL}=$ high-density lipoprotein 
Table 2. Comparison of data by number of relapses

\begin{tabular}{lccc}
\hline & $\begin{array}{c}\text { Group 1 } \\
\mathbf{3} \text { relapses } \\
\mathbf{n = 4 3}\end{array}$ & $\begin{array}{c}\text { Group 2 } \\
\mathbf{3} \text { relapses } \\
\mathbf{n = 2 7}\end{array}$ & $\boldsymbol{p}$ value \\
& $10 / 33$ & $12 / 15$ & \\
\hline Male/ female & $37.20 \pm 10.13$ & $40.88 \pm 9.36$ & 0.063 \\
Age, years & $2.51 \pm 1.68$ & $3.25 \pm 1.62$ & 0.133 \\
\hline EDSS & $6.02 \pm 5.36$ & $9.85 \pm 6.01$ & 0.072 \\
Disease duration, years & & & $\mathbf{0 . 0 0 7}$ \\
Number of lesions & 17 & 6 & 0.133 \\
\multicolumn{1}{c}{ 1-9 Lesions } & 26 & 21 & \\
Total cholesterol, mg/dL & $177.81 \pm 37.66$ & $230.48 \pm 72.59$ & $<\mathbf{0 . 0 0 1}$ \\
Triglycerides, mg/dL & $118.02 \pm 79.41$ & $159.89 \pm 89.87$ & $\mathbf{0 . 0 4 5}$ \\
\hline HDL, $\mathrm{mg} / \mathrm{dL}$ & $51.88 \pm 15.59$ & $54.02 \pm 15.06$ & 0.573 \\
\hline LDL, $\mathrm{mg} / \mathrm{dL}$ & $102.42 \pm 32.21$ & $139.40 \pm 56.81$ & $\mathbf{0 . 0 0 1}$ \\
\hline
\end{tabular}

Data are shown mean \pm standard deviation or number. EDSS $=$ Expanded Disability Status Scale, LDL $=$ lowdensity lipoprotein, HDL = high-density lipoprotein

lesions and $>9$ lesions according to the number of lesions observed on cranial MRI and the data were compared. There was no significant difference between the groups in terms of gender, duration of disease and lipid parameters, whereas patients were older ( $40.35 \pm 10.15$ years vs. $35.08 \pm 8.65$ years) and EDSS scores were higher in $>9$ lesion group (Table $3)$.

Finally, in the correlation analysis, there was a significant positive correlation between total cholesterol and LDL levels and EDSS scores and disease duration (Table 4).

\section{DISCUSSION}

In this study, we found a significant correlation between the total number of relapses and cholesterol levels. There was a positive correlation between disease duration and EDSS scores and total cholesterol and LDL levels. Relapses in MS are the result of ongoing inflammatory process and disease activation. As a result of demyelination and neurodegeneration in axons during relapses, a number of degradation products are formed in the peripheral blood. The focus of most of ongoing studies is to detect these

Table 3. Comparison of data by number of lesions

\begin{tabular}{lccc}
\hline & $\begin{array}{c}\text { Lesion } \leq \mathbf{9} \\
\mathbf{n}=\mathbf{2 3}\end{array}$ & $\begin{array}{c}\text { Lesion }>\mathbf{9} \\
\mathbf{n}=\mathbf{4 7}\end{array}$ & $\boldsymbol{p}$ value \\
\hline Male/ female & $6 / 17$ & $16 / 31$ & 0.501 \\
Age, years & $35.08 \pm 8.65$ & $40.36 \pm 10.15$ & $\mathbf{0 . 0 3 6}$ \\
EDSS & $1.69 \pm 0.82$ & $3.34 \pm 1.75$ & $\mathbf{0 . 0 0 0}$ \\
Disease duration, years & $5.82 \pm 5.20$ & $8.31 \pm 6.08$ & 0.096 \\
Total cholesterol, mg/dL & $188.39 \pm 66.22$ & $202.89 \pm 55.73$ & 0.340 \\
Triglycerides, $\mathrm{mg} / \mathrm{dL}$ & $116.18 \pm 77.14$ & $142.96 \pm 88.70$ & 0.221 \\
HDL, $\mathrm{mg} / \mathrm{dL}$ & $53.01 \pm 17.22$ & $52.56 \pm 14.48$ & 0.908 \\
\hline LDL, $\mathrm{mg} / \mathrm{dL}$ & $111.59 \pm 50.89$ & $119.18 \pm 44.79$ & 0.527 \\
\hline D & &
\end{tabular}

Data are shown mean \pm standard deviation or number. EDSS $=$ Expanded Disability Status Scale, LDL $=$ lowdensity lipoprotein, $\mathrm{HDL}=$ high-density lipoprotein 
Table 4. Evaluation of the correlation analysis between EDSS and disease duration and lipid parameters

\begin{tabular}{lcc}
\hline & r & $\boldsymbol{p}$ value \\
\hline Tchol/ EDSS & 0.283 & $\mathbf{0 . 0 1 8}$ \\
LDL/ EDSS & 0.346 & $\mathbf{0 . 0 0 3}$ \\
\hline HDL/ EDSS & 0.008 & 0.945 \\
Trig/ EDSS & 0.130 & 0.285 \\
Tchol/ Disease duration & 0.293 & $\mathbf{0 . 0 1 4}$ \\
LDL/ Disease duration & 0.327 & $\mathbf{0 . 0 0 6}$ \\
HDL/ Disease duration & -0.067 & 0.582 \\
Trig/ Disease duration & 0.215 & 0.074 \\
\hline
\end{tabular}

Tchol $=$ Total cholesterol, EDSS $=$ Expanded Disability Status Scale, LDL = low-density lipoprotein, HDL = highdensity lipoprotein, Trig $=$ Triglyserides

degradation products. Forty percent of the myelin sheath consists of water, while the remainder consists of lipids $(75 \%)$ and proteins $(25 \%)$. In experimental models of encephalomyelitis, increased concentrations of cholesterol esters, sulfatide and cerebrosides have been shown in CNS during myelin destruction [4]. Consistent with the literature, our findings support increased cholesterol in peripheral blood as a result of increased myelin destruction due to increased number of relapses under longer disease duration.

Eighty percent of plasma $24 \mathrm{~S}-\mathrm{OH}$-cholesterol which is thought to be an indicator of brain cholesterol degradation originates from the brain. Studies have shown a significant positive correlation between serum cholesterol and 24S-OH-cholesterol [5-7]. It has been shown that after $24 \mathrm{~S}-\mathrm{OH}$-cholesterol was formed, it was crossed the blood-brain barrier and was transported in plasma by low-density lipoprotein (LDL) [8]. Increased plasma cholesterol levels may reflect neurodegeneration and demyelination processes in MS patients.

Researchers have found links between cholesterol metabolism, inflammatory pathways and the immune system. The best example of this is the removal of cholesterol crystals by macrophages in atherosclerosis [9]. Proinflammatory properties of lipids are thought to be effective in the pathogenesis of MS. Dyslipidemia can induce inflammation that causes dysfunction of the endothelium by initiating upregulation of adhesion molecules and aggregation of monocytes. The passage of immune cells through the blood brain barrier via the vascular endothelium constitutes one of the most important phases of MS pathogenesis $[10,11]$. LDL particles in the serum of MS patients have been shown to be more susceptible to oxidation. These particles are also thought to be proinflammatory because they have less affinity for LDL receptors [12]. MS patients have been found to have high oxidized LDL in plasma and CSF samples, and have high levels of autoantibodies against them in their sera [13-15]. HDL has an anti-inflammatory effect by inhibiting the expression of adhesion molecules in endothelial cells and by reducing the flow of monocytes. High HDL values have been found to be associated with lower injury and cell extravasation in the blood brain barrier $[16,17]$.

Although cholesterol metabolism in the brain is independent, inflammation and oxidative stress in the body can affect brain function and contribute to increased activity in MS. Therefore, it is not known exactly whether high lipid levels in the blood cause deterioration, or whether increased lipid levels are due to increased myelin destruction associated with increased disease activity [2].

In a study evaluating MS patients with a single relapse, a significant correlation was found between the mean number of contrast-enhancing lesions and total cholesterol and LDL cholesterol levels [18]. In another study, high LDL cholesterol levels were shown to correlate with the number of new T2 lesions and the total number of new / enlarging T2 lesions. Weinstein-Guttman et al. [19] demonstrated the adverse effect of lipid profile on MRI lesion activity of high-risk patient who experience their first relapse. While age and EDSS scores were significantly correlated with the number of lesions, cholesterol levels didn't show any differences in our study.

In the literature, it has been shown that EDSS score was correlated with total cholesterol levels, and the increase in total cholesterol and triglyceride levels were correlated with increased disability scores [3]. In another study with a larger sample size, it was shown that total cholesterol and triglyceride levels were lower in patients with slow progression than in those with rapid progression. Based on this, it is thought that 
serum lipid profile may have an effect on disease progression. In the same study, a positive correlation was found between total cholesterol levels and EDSS score in MS patients [2]. Similarly, we found a positive correlation between EDSS score and total cholesterol and LDL levels, but no significant correlation with triglyceride and HDL levels.

\section{Limitations}

The limited number of cases, lack of control group, unregistered body mass indices, cross-sectional examination of MRI, and the absence of distinction of emerging and contrast-enhancing lesions may be considered as limitations of our study.

\section{CONCLUSION}

In conclusion, we found that the number of relapses, duration of disease and EDSS scores were significantly correlated significantly with cholesterol levels. Increased plasma cholesterol levels may be detected as a result of increased metabolism as a result of myelin destruction in the CNS that occurs during the relapse and long disease course. In the follow-up of MS patients, changes in plasma cholesterol levels which are an easily accessible laboratory test may provide insight into disease activity and progression. Further studies in larger samples of MS patients are needed to understand this relationship.

\section{Conflict of interest}

The authors disclosed no conflict of interest during the preparation or publication of this manuscript.

\section{Financing}

The authors disclosed that they did not receive any grant during conduction or writing of this study.

\section{REFERENCES}

1. Comabella M, Montalban X. Body fluid biomarkers in multiple sclerosis. Lancet Neurol 2014;13:113-26.

2. Durfinova M, Prochazkova L, Petrlenicova D, Bystricka Z, Oresanska K, Kuracka L, et al. Cholesterol level correlate with disability score in patients with relapsing-remitting form of multiple sclerosis. Neurosci Lett. 2018;687:304-7.
3. Zhornitsky S, McKay KA, Metz LM, Teunissen CE, Rangachari M. Cholesterol and markers of cholesterol turnover in multiple sclerosis: relationship with disease outcomes. Mult Scler Relat Disord 2016;5:53-65.

4. Maggio B, Cumar FA. Experimental allergic encephalomyelitis: dissociation of neurological symptoms from lipid alterations in brain. Nature 1975;253:364-5.

5. Bjorkhem I, Lutjohann D, Diczfalusy U, Stahle L, Ahlborg G, Wahren J. Cholesterol homeostasis in human brain: turnover of 24S-hydroxycholesterol and evidence for a cerebral origin of most of this oxysterol in the circulation. J Lipid Res 1998;39:1594-600.

6. Leoni V, Masterman T, Diczfalusy U, De Luca G, Hillert J, Bjorkhem I. Changes in human plasma levels of the brain specific oxysterol 24S-hydroxycholesterol during progression of multiple sclerosis. Neurosci Lett 2002;331:163-6.

7. Teunissena CE, Dijkstra CD, Polman CH, Hoogervorst ELJH, von Bergman K, Lütjohann D. Decreased levels of the brain specific 24S-hydroxycholesterol and cholesterol precursors in serum of multiple sclerosis patients. Neurosci Lett 2003;347:15962.

8. Vega GL, Weiner MF, Lipton AM, von Bergmann $\mathrm{K}$, Lütjohann D, Moore C, et al. Reduction in levels of 24Shydroxycholesterol by statin treatment in patients with Alzheimer disease. Arch Neurol 2003;60:510-5.

9. Tall AR, Yvan-Charvet L. Cholesterol, inflammation and innate immunity. Nat Rev Immunol 2015;15:104-16.

10. Sitia S, Tomasoni L, Atzeni F, Ambrosio G, Cordiano C, Catapano A, et al. From endothelial dysfunction to atherosclerosis. Autoimmun Rev 2010;9:830-4.

11. Frohman EM, Racke MK, Raine CS. Multiple sclerosis--the plaque and its pathogenesis. N Engl J Med 2006;354:942-55.

12. Schmitz G, Grandl M. Role of redox regulation and lipid rafts in macrophages during Ox-LDL-mediated foam cell formation. Antioxid Redox Signal 2007;9:1499-518.

13. Newcombe J, Li H, Cuzner ML. Low density lipoprotein uptake by macrophages in multiple sclerosis plaques: implications for pathogenesis. Neuropathol Appl Neurobiol 1994;20:152-62.

14. Palavra F, Marado D, Mascarenhas-Melo F, Sereno J, Teixeira-Lemos E, Nunes CC, et al. New markers of early cardiovascular risk in multiple sclerosis patients: oxidized-LDL correlates with clinical staging. Dis Markers 2013;34:341-8.

15. Besler HT, Comoglu S. Lipoprotein oxidation, plasma total antioxidant capacity and homocysteine level in patients with multiple sclerosis. Nutr Neurosci 2003;6:189-96.

16. Fellows K, Uher T, Browne RW, Weinstock-Guttman B, Horakova D, Posova H, et al. Protective associations of HDL with blood-brain barrier injury in multiple sclerosis patients. $\mathrm{J}$ Lipid Res 2015;56:2010-8.

17. Jorissen W, Wouters E, Bogie JF, Vanmierlo T, Noben JP, Sviridov D, et al. Relapsing-remitting multiple sclerosis patients display an altered lipoprotein profile with dysfunctional HDL. Sci Rep 2017;7:43410.

18. Giubilei F, Antonini G, Di Legge S, Sormani MP, Pantano P, Antonini R, et al. Blood cholesterol and MRI activity in first 
clinical episode suggestive of multiple sclerosis. Acta Neurol Scand 2002;106:109-12.

19. Weinstock-Guttman B, Zivadinov R, Horakova D, Havrdova

E, Qu J, Shyh G, et al. Lipid profiles are associated with lesion formation over 24 months in interferon-beta treated patients following the first demyelinating event. J Neurol Neurosurg Psychiatry 2013;84:1186-91. 